\title{
Lipid Alterations in African American Prostate Cancer
}

Anindita Ravindran ${ }^{1 \ddagger}$, Danthasinghe Waduge Badrajee Piyarathna ${ }^{1 \ddagger}$, Jie Gohlke ${ }^{1 \ddagger}$, Vasanta Putluri $^{2}$, Tanu Soni ${ }^{3}$, Stacy Lloyd ${ }^{1,4}$, Patricia Castro ${ }^{5,10}$, Subramaniam Pennathur ${ }^{6}$, Jeffrey A Jones $^{4,7}$, Michael Ittmann ${ }^{4.5}$, Nagireddy Putluri ${ }^{1,2,4}$, George Michailidis ${ }^{8 \#}$, Thekkelnaycke M Rajendiran $^{3,9, \#}$, Arun Sreekumar ${ }^{1,4, \#, *}$

${ }^{1}$ Department of Molecular and Cellular Biology, and Center for Metabolism and Experimental Therapeutics, Baylor College of Medicine, One Baylor Plaza, Houston TX 77030.

${ }^{2}$ Advanced Technology Core, Baylor College of Medicine, One Baylor Plaza, Houston TX 77030.

${ }^{3}$ Michigan Regional Comprehensive Metabolomics Resource Core, University of Michigan, Ann Arbor, MI-48105.

${ }^{4}$ Dan L Duncan Comprehensive Cancer Center, Baylor College of Medicine, One Baylor Plaza, Houston TX 77030.

${ }^{5}$ Department of Pathology and Immunology, Baylor College of Medicine, One Baylor Plaza, Houston TX 77030.

${ }^{6}$ Division of Nephrology, Department of Medicine, University of Michigan, Ann Arbor, MI48105 and Department of Molecular and Integrative Physiology, University of Michigan, Ann Arbor, Michigan, USA 84105

${ }^{7}$ Department of Urology, Baylor College of Medicine, One Baylor Plaza, Houston TX 77030 and Urology Clinic, Michael E Debakey Veterans Affairs Medical Center, Houston, TX 77030.

${ }^{8}$ Informatics Institute, University of Florida, Gainesville, FL 32611

${ }^{9}$ Department of Pathology and Michigan Center for Translational Pathology, University of Michigan, Ann Arbor-MI-48105.

${ }^{10}$ Human Tissue Acquisition \& Pathology Shared Resource, Dan L Duncan Comprehensive Cancer Center, Baylor College of Medicine, Houston, TX 77030. 
$\$$ Equal First Authors

${ }^{\#}$ Equal Senior Authors

\section{*Correspondence}

Arun Sreekumar, Ph.D.

Charles C Bell Endowed Professor

Center for Metabolism and Experimental Therapeutics

Department of Molecular and Cell Biology

Baylor College of Medicine

Rm 120 DJ, Jewish Biomedical Research Building

One Baylor Plaza

Houston, TX-77030

Email: Arun.Sreekumar@bcm.edu

\section{Disclosures}

The authors have no conflicts of interest to disclose.

\section{Funding}

This research was partially funded by National Cancer Institute grant numbers RO1CA227559 and

RO1CA227559S1 (ASK), Prostate Cancer Foundation Challenge Award (ASK, JAJ, MI) P30CA125123 Metabolomics Shared Resources (ASK, NP), P30CA125123 Integrated Biospecimen Shared Resource (MI), R01CA220297 (NP), P30DK089503 and P30DK081943 
(SP), and partially supported by P50CA186784 (ASK); CPRIT RP17005 and RP210227 to the

Proteomics and Metabolomics Core Facility (ASK and NP), Alkek Center for Molecular Discovery (ASK) and Agilent Technologies Center for Excellence in Mass Spectrometry (ASK). Dr. Sreekumar is supported by Charles C Bell Jr. Endowment from Baylor College of Medicine.

\section{Author Contributions}

A.S.K and G.M. designed the study. A.S.K. supervised the study. J.G. and V.P. performed the mass spectrometry experiments. S.T, T.M.R, S.P and N.P. helped develop the lipidomics platform and associated data analysis pipeline, D.W.B.P. and S.L. analyzed the data, M.I., P.C., and J.A.J contributed clinical samples and clinical insights; A.R., S.L., J.G., J.A.J., S.P., S.L., G.M., A.S.K. provided inputs for data interpretation; D.W.B.P. and A.R. generated the figures; A.R., J.G., G.M., and A.S.K. wrote the paper. 


\begin{abstract}
African-American (AA) men are more than twice as likely to die of prostate cancer (PCa) than European American (EA) men. Previous in-silico analysis revealed enrichment of altered lipid metabolic pathways in pan-cancer AA tumors. Here, we performed global unbiased lipidomics profiling on 48 matched localized PCa and benign adjacent tissues (30 AA, 24 ancestry-verified, and 18 EA, 8 ancestry verified) and quantified 429 lipids belonging to 15 lipid classes. Significant alterations in long chain polyunsaturated lipids was observed between PCa and benign adjacent tissues, low and high Gleason tumors, as well as associated with early biochemical recurrence, both in the entire cohort, and within AA patients. Altered levels of cholesteryl esters, and phosphatidyl inositols delineated AA and EA PCa, while levels of triglycerides, phosphatidyl glycerol, phosphatidyl choline, phosphatidic acid and cholesteryl esters distinguished AA and EA PCa patients with biochemical recurrence. These first-in-field results implicate lipid alterations as biological factors for prostate cancer disparities.
\end{abstract}

\title{
Keywords
}

Lipidomics, Prostate Cancer Health Disparity, Biochemical Recurrence, Cholesteryl Esters

\section{Introduction}

Prostate cancer $(\mathrm{PCa})$ progression is twice as aggressive and more than twice as morbid in African Americans (AA) compared to European Americans (EA), yet the specific biochemical basis underlying this disparity remains unknown ${ }^{1}$. While environmental factors such as diet and access to proper healthcare may be significant causes for increased mortality rates associated with PCa in AA men, recent reports suggest that metabolic alterations may also drive disparity in PCa progression $^{2}$. Our previous in silico study described enriched adipogenesis, fatty acid metabolism 
and cholesterol homeostasis, among others, as being enriched in AA tumors across multiple different tumor types, which was further validated in an independent PCa gene expression dataset ${ }^{3}$. In addition, elevated cholesterol levels in serum have been reported to be associated with increased biochemical recurrence (BCR) specifically in AA men with $\mathrm{PCa}^{4}$. Here, for the first time we describe race-specific clinically relevant alterations in PCa lipidome.

\section{Results}

We performed unbiased global lipidomic profiling using Triple Time Of Flight (Triple-TOF) liquid chromatography-mass spectrometry (LC-MS) on 48 localized PCa and matched benign adjacent tissue (Table 1 for Clinical Information, 30AA and 18 EA pairs, 24 AA and 8 EA tissues were ancestry verified). The profiling platform was highly reproducible with a $\% \mathrm{CV}$ of $2.7 \%$ in the liver pool $(n=24)$ for 429 lipids measured, CV of $5.3 \%$ for internal standards $(n=26$, Supplementary Figure 1) and normal distribution of the lipids overall and within each class (Supplementary Figure 2). The 429 lipid species identified belonged to 15 lipid classes including cholesteryl esters (CE), diacylglycerols (DG), lysophosphatidyl cholines (L-PC), lysophosphatidyl ethanolamines (L-PE), phosphatidic acid (PA), phosphatidyl cholines (PC), phosphatidyl ethanolamines (PE), phosphatidyl glycerol (PG), phosphatidyl inositols (PI), plasmenylphosphatidyl cholines (P-PC), plasmenyl-phosphatidyl ethanolamines (P-PE), phosphatidyl serines (PS), sphingomyelins (SM), and triglycerides (TG) (Supplementary Tables 1-3).

Principal Component Analysis (PCA) using all the lipids revealed a moderate separation of the benign and cancer samples, both across the entire cohort and within AA patients, suggestive of alterations in lipid components between these two tissue types (Figure 1A and Supplementary 
Figure 3A). Similarly, across the entire cohort as well as with AA patients, comparing PCa with benign adjacent tissue, the significantly altered lipids (FDR $<0.1)$ within each lipid class were predominantly polyunsaturated ( $>2$ double bonds) with long chain fatty acid chains (20-40 fatty acids, Figures 1B, C and Supplementary Figures 3B, C, 4 and 5) for altered lipids with FDR < 0.25). Included here were prominent elevation in levels of CE, TG and reduced levels of SM, PS, P-PE, PI and PG (Figure 1C and Supplementary Figure 3C, Supplementary Figures 4 and 5, Supplementary Tables 1 and 2). In addition, reduced levels of L-PC (across all PCa and within AA PCa), and SM (only within AA PCa), and significantly elevated levels of TG (only within AA PCa) were significantly associated with high Gleason tumors (Gleason $>7$ or $=4+3$, Figure $1 D$ and Supplementary Figure 3D, all FDR<0.25). Further, across all PCa, lower levels of L-PC, SM, and PE were significantly associated with early biochemical recurrence (BCr, 5 years postprostatectomy, FDR<0.35), a clinical indicator of aggressive PCa (Supplementary Figure 3D). Similarly, reduced levels of L-PC and P-PC, and elevated levels of PI were associated with early BCr with AA PCa patients (FDR<0.35, Figure 1E).

Intriguingly, there were key changes in lipid profiles comparing Gleason grade matched AA $(n=30)$ and EA $(n=18)$ tumors. As shown in Figure 2A, PCA analysis of the lipid profiles was able to delineate AA and EA tumors for the most part. Furthermore, lipids (predominantly polyunsaturated and long chain) belonging to 10 lipid classes significantly distinguished AA and EA PCa (Figure 2B, Supplementary Figure 6A), that included prominent elevation (FDR < 0.1) in levels of CE, TG, PI and PA class of lipids (Figure $\mathbf{2 C}$, altered lipids with FDR $<0.25$ in Supplementary Figure 6, Supplementary Table 3). Interestingly, comparison of AA and EA PCa patients with early $\mathrm{BCr}$ revealed conspicuous elevation (FDR $<0.35$, altered at least two-fold) 
in levels of TG and CE, in the former, suggesting race-specific de-regulation of different lipid pathways in aggressive tumors (Figure 2D).

\section{Discussion}

Our finding describes unique race-associated alterations in specific lipid classes that include increase in CEs, PI, and TGs in PCa. Interestingly, both CE and TGs were prominently higher in AA vs EA patients with early BCr.

CEs are the primary source of cholesterol for steroidogenesis ${ }^{5}$. CEs (cholesterol oleate in particular) have been detected in a previous lipidomic screen and proposed as potential molecular biomarkers for $\mathrm{PCa}^{6}$. Increases in CEs were also shown to be associated with $\mathrm{PCa}$ tumor progression and metastasis and aberrant accumulation of CEs was found to be correlated with PTEN loss and PI3K/Akt activation ${ }^{7,8}$. This activation of the PI3K/Akt pathway, which has recently been demonstrated to be higher in $\mathrm{AA} \mathrm{PCa}$, is corroborated by elevated PI (an important second messenger in the PI3K/Akt pathway) levels in these tumors in our analysis. Furthermore, this is consistent with our previous publication where we reported that the oncogene MNX1 is upregulated in AA men in response to activated PI3K/AKT signaling and drives lipid synthesis via activation of SREBP1 and fatty acid synthase ${ }^{9}$.

In addition, high levels of TGs seen in AA PCa are thought to be associated with PCa progression $^{10}$. TGs are an important energy source when hydrolyzed into free fatty acids (FFAs). Cancer cells use the energy generated from these TG pools to drive tumor proliferation, specifically under hypoxia ${ }^{11}$. 
Intriguingly, increase in $\mathrm{CE}$ and $\mathrm{TG}$ in $\mathrm{AA}$ vs $\mathrm{EA}$ patients with early $\mathrm{BCr}$ is a novel finding that could be regulated by androgens that are known to affect triglyceride and cholesteryl ester pools by contributing to lipid synthesis ${ }^{12}$.

Interestingly, in our study, the majority of the altered lipids in the context of race in PCa include polyunsaturated fatty acids (PUFAs). PUFAs are known to be high in AA diet which predominantly comprises of corn and soy ${ }^{13}$. Moreover, AA men are known to have significantly higher circulating levels of arachidonic acid (a long chain PUFA implicated in inflammation) than EA men ${ }^{14}$. Our finding that the AA PCa lipidome is rich in long chain PUFAs further supports these prior observations and potentially reveals an impact of diet on PCa risk or progression.

The high incidence of PCa as well as the aggressive nature of tumor progression in AA men make it imperative to seek novel biological means to comprehend the disparity. Understanding the alterations in lipid composition and thereby discovering lipid biomarkers that are prognostic for PCa progression in AA but not EA men may be critical to developing race-associated therapeutic strategies. Our finding of increased CEs and TGs in AA PCa strongly supports the use of these specific lipid classes as prognostic biomarkers for aggressive PCa progression.

Taken together, our descriptive data for the first time reveals key changes in PCa lipidome in AA compared to EA patients. Additional studies are needed to address these key findings in the context of PCa disparities. 


\section{Methods:}

Lipid extraction from tissues and mass spectrometry analysis were performed as described previously ${ }^{15-18}$. Missing values were imputed using the K nearest-neighbor method (KNN). Data was $\log 2$ transformed and day median normalized and checked to ensure normality, both with in each lipid class and across all lipids measured (Supplementary Figure 2). Unless specified, differential lipids were determined using paired t-test $(\mathrm{p}<0.05)$, followed by the BenjaminiHochberg $(\mathrm{BH})$ procedure for false discovery rate (both FDR $<0.1$ and FDR $<0.25$ have been shown) correction. Lipid profiles comparing AA vs EA PCa was determined by subtracting their respective paired benign values, and using a permutation test ( $\mathrm{p}<0.05$, FDR<0.25). Mann-Whitney statistics with BH FDR correction was used to determine lipid classes that distinguished PCa by Gleason and $\mathrm{BCr}$. FDR $<0.25$ and $\mathrm{FDR}<0.35$, respectively were used to determine altered lipids in comparisons involving Gleason and $\mathrm{BCr}$.

The normalized data for this study has been deposited to the Metabolomics Workbench (https://www.metabolomicsworkbench.org/) under the trackID 2901.

\section{References}

1. IJ P. Epidemiology and pathophysiology of prostate cancer in African-American men. The Journal of urology. 2007;177(2):444-449. doi:10.1016/J.JURO.2006.09.024

2. JH G, SM L, S B, et al. Methionine-Homocysteine Pathway in African-American Prostate Cancer. JNCI cancer spectrum. 2019;3(2). doi:10.1093/JNCICS/PKZ019

3. DWB P, A B, JM A, et al. ERR1 and PGC1 $\alpha$ associated mitochondrial alterations correlate with pan-cancer disparity in African Americans. The Journal of clinical investigation. 2019;129(6):2351-2356. doi:10.1172/JCI127579

4. EH A, LE H, WJ A, et al. Racial Differences in the Association Between Preoperative Serum Cholesterol and Prostate Cancer Recurrence: Results from the SEARCH Database. Cancer epidemiology, biomarkers \& prevention : a publication of the American Association 
for Cancer Research, cosponsored by the American Society of Preventive Oncology. 2016;25(3):547-554. doi:10.1158/1055-9965.EPI-15-0876

5. FB K, VK K, WJ S, S A. Cholesterol ester droplets and steroidogenesis. Molecular and cellular endocrinology. 2013;371(1-2):15-19. doi:10.1016/J.MCE.2012.10.012

6. J L, S R, HL P, et al. Integration of lipidomics and transcriptomics unravels aberrant lipid metabolism and defines cholesteryl oleate as potential biomarker of prostate cancer. Scientific reports. 2016;6. doi:10.1038/SREP20984

7. S Y, J L, SY L, et al. Cholesteryl ester accumulation induced by PTEN loss and PI3K/AKT activation underlies human prostate cancer aggressiveness. Cell metabolism. 2014;19(3):393-406. doi:10.1016/J.CMET.2014.01.019

8. HJ L, J L, RE V, et al. Cholesterol Esterification Inhibition Suppresses Prostate Cancer Metastasis by Impairing the Wnt/ $\beta$-catenin Pathway. Molecular cancer research : MCR. 2018;16(6):974-985. doi:10.1158/1541-7786.MCR-17-0665

9. L Z, J W, Y W, et al. MNX1 Is Oncogenically Upregulated in African-American Prostate Cancer. Cancer research. 2016;76(21):6290-6298. doi:10.1158/0008-5472.CAN-16-0087

10. L W, M J, S H, et al. Hypertriglyceridemia as a possible risk factor for prostate cancer. Prostate cancer and prostatic diseases. 2005;8(4):316-320. doi:10.1038/SJ.PCAN.4500834

11. IR S, DK N, A R, et al. Hypoxia induces triglycerides accumulation in prostate cancer cells and extracellular vesicles supporting growth and invasiveness following reoxygenation. Oncotarget. 2015;6(26):22836-22856. doi:10.18632/ONCOTARGET.4479

12. JV S, M E, K G, W H, G V. Androgens stimulate fatty acid synthase in the human prostate cancer cell line LNCaP. Cancer research. 1997;57(6):1086-1090. Accessed October 25, 2021. https://pubmed.ncbi.nlm.nih.gov/9067276/

13. FH C, RC M, BA W, et al. Diet-gene interactions and PUFA metabolism: a potential contributor to health disparities and human diseases. Nutrients. 2014;6(5):1993-2022. doi:10.3390/NU6051993

14. RA M, S S, I R, et al. The impact of FADS genetic variants on $\omega 6$ polyunsaturated fatty acid metabolism in African Americans. BMC genetics. 2011;12. doi:10.1186/1471-2156$12-50$

15. C B, M AV, T Z, W K, F C. "Bligh and Dyer" and Folch Methods for Solid-Liquid-Liquid Extraction of Lipids from Microorganisms. Comprehension of Solvatation Mechanisms and towards Substitution with Alternative Solvents. International journal of molecular sciences. 2017;18(4). doi:10.3390/IJMS18040708

16. V V, J D, CR A, et al. Multi-omics Integration Analysis Robustly Predicts High-Grade Patient Survival and Identifies CPT1B Effect on Fatty Acid Metabolism in Bladder Cancer. 
Clinical cancer research: an official journal of the American Association for Cancer Research. 2019;25(12):3689-3701. doi:10.1158/1078-0432.CCR-18-1515

17. P P, F G, DWB P, et al. Unbiased Lipidomic Profiling of Triple-Negative Breast Cancer Tissues Reveals the Association of Sphingomyelin Levels with Patient Disease-Free Survival. Metabolites. 2018;8(3). doi:10.3390/METABO8030041

18. DWB P, TM R, V P, et al. Distinct Lipidomic Landscapes Associated with Clinical Stages of Urothelial Cancer of the Bladder. European urology focus. 2018;4(6):907-915. doi:10.1016/J.EUF.2017.04.005 


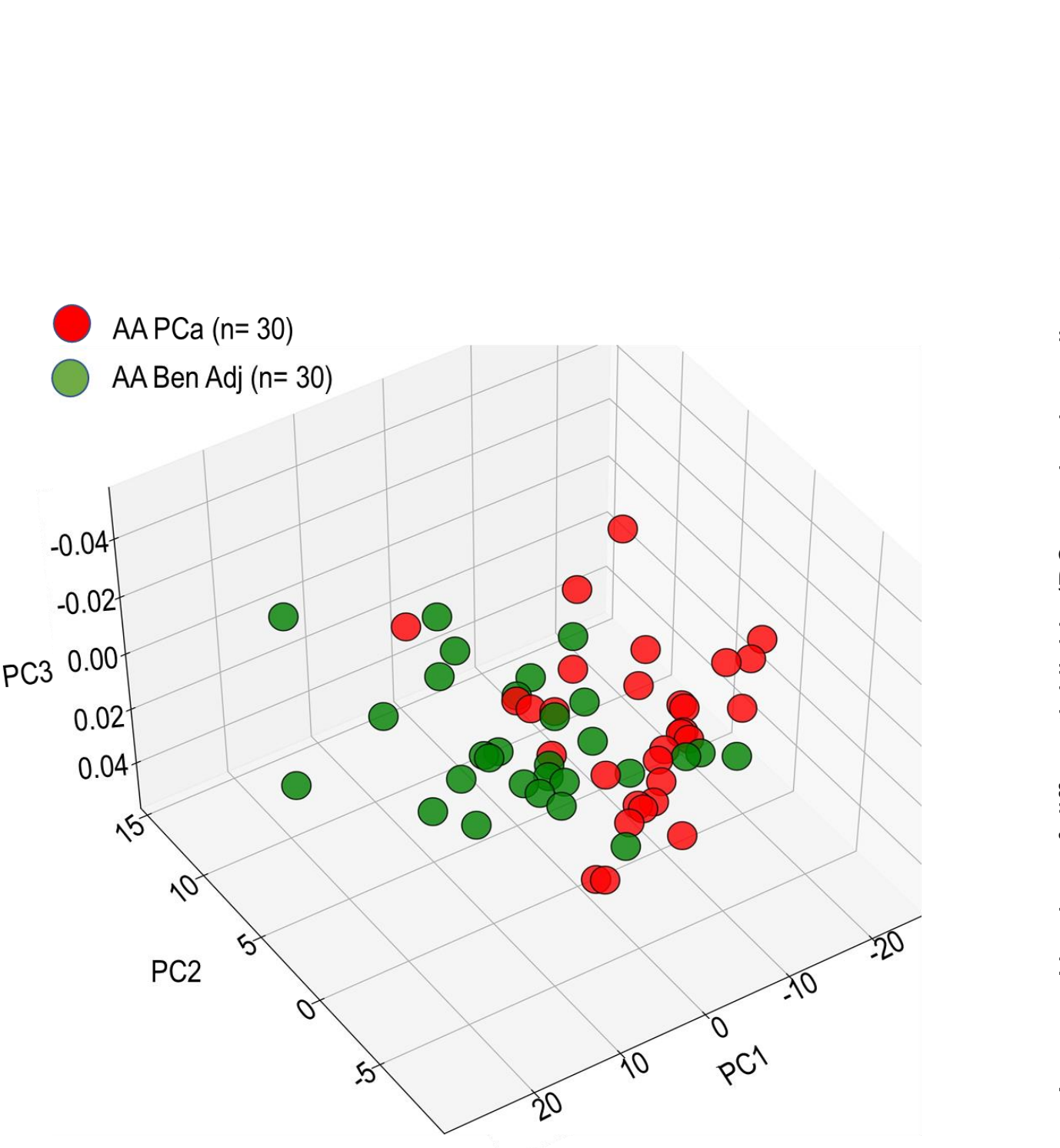

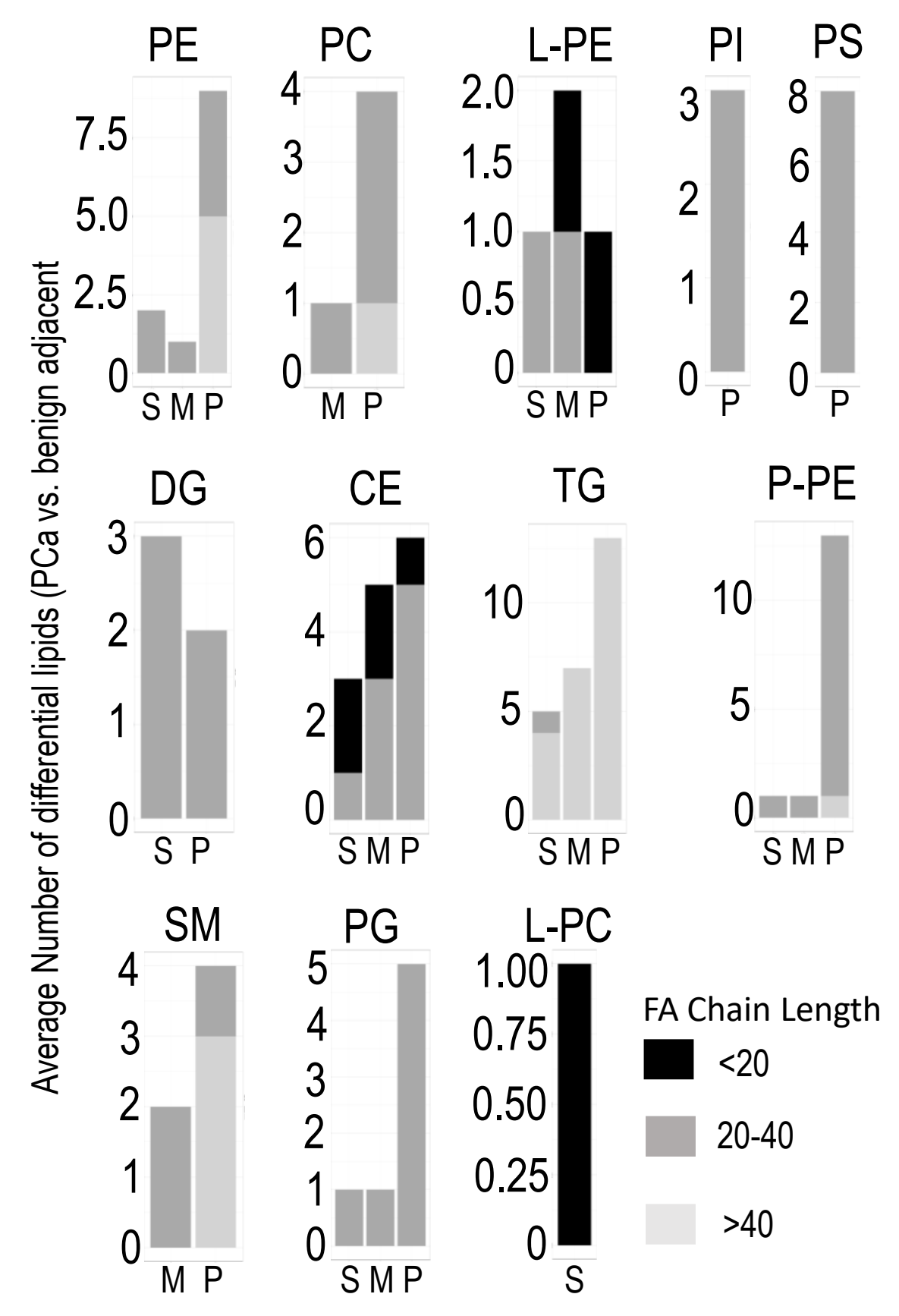

C

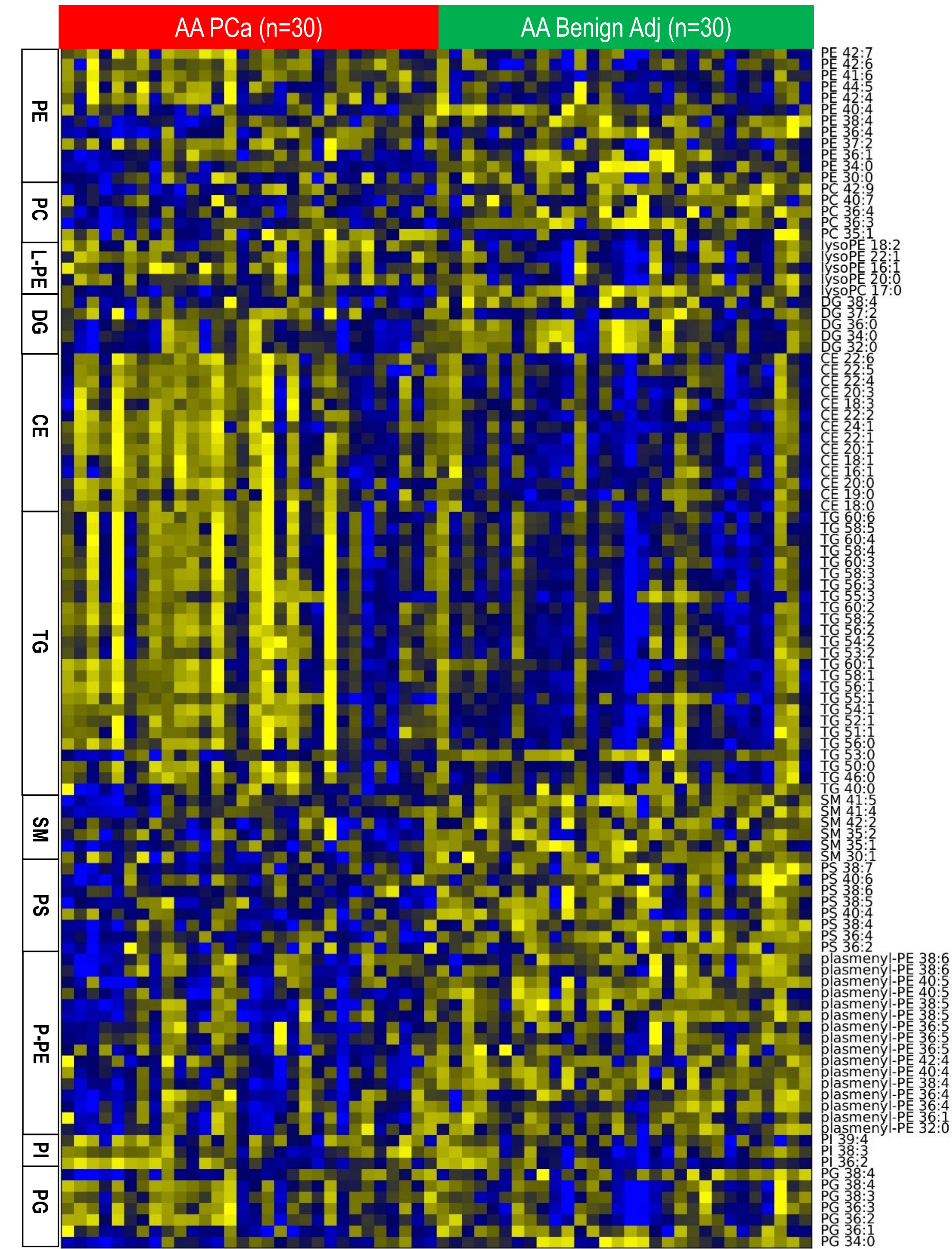

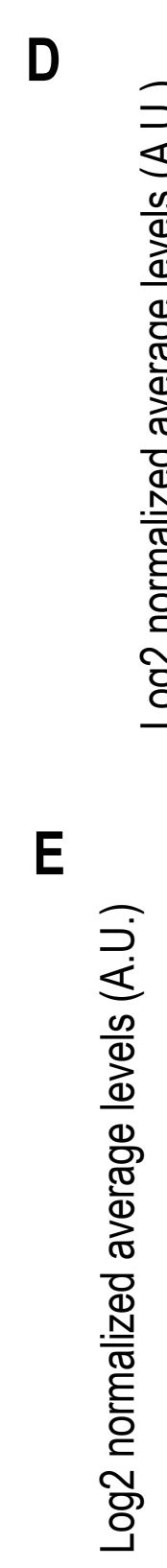
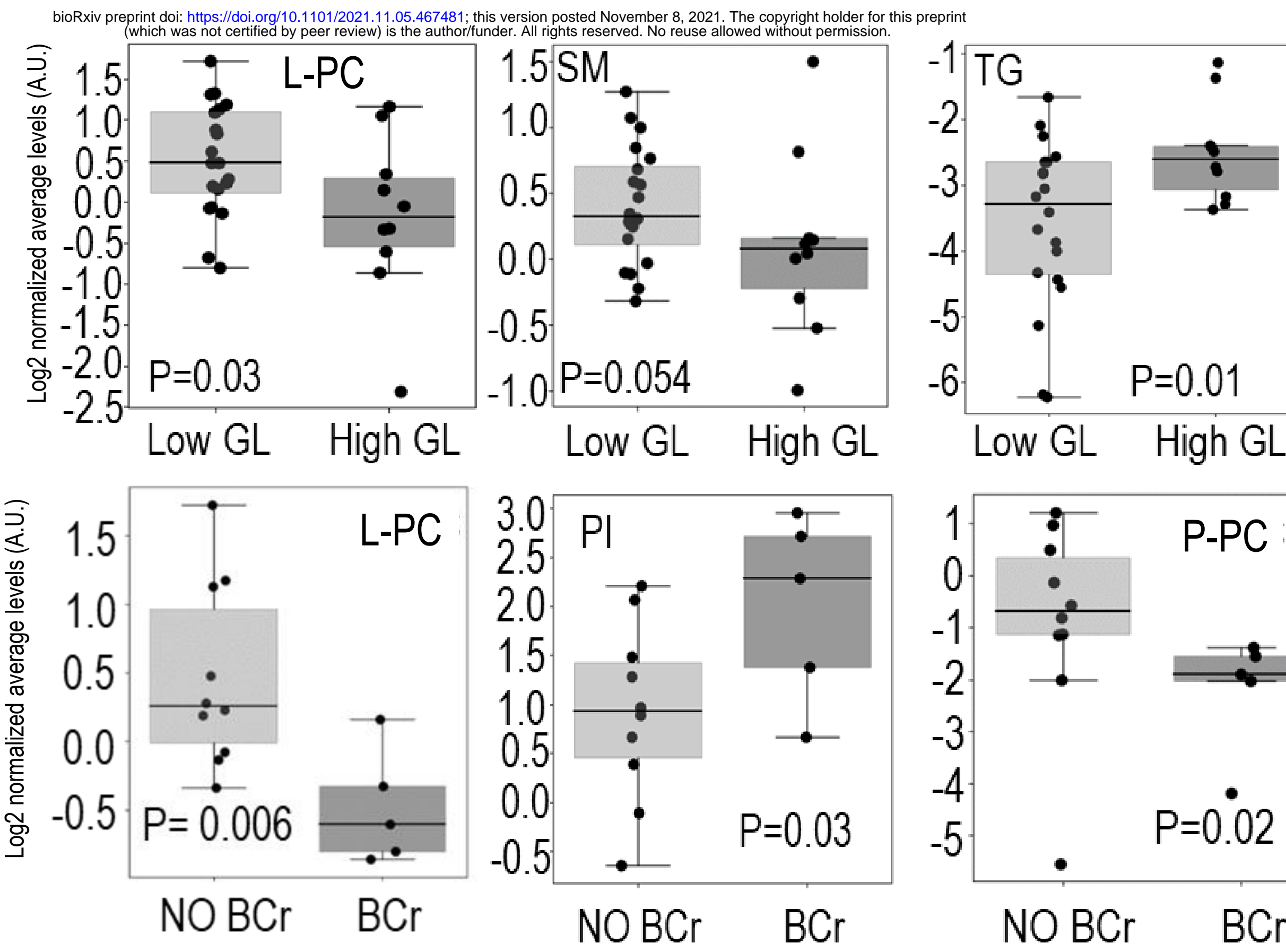
A
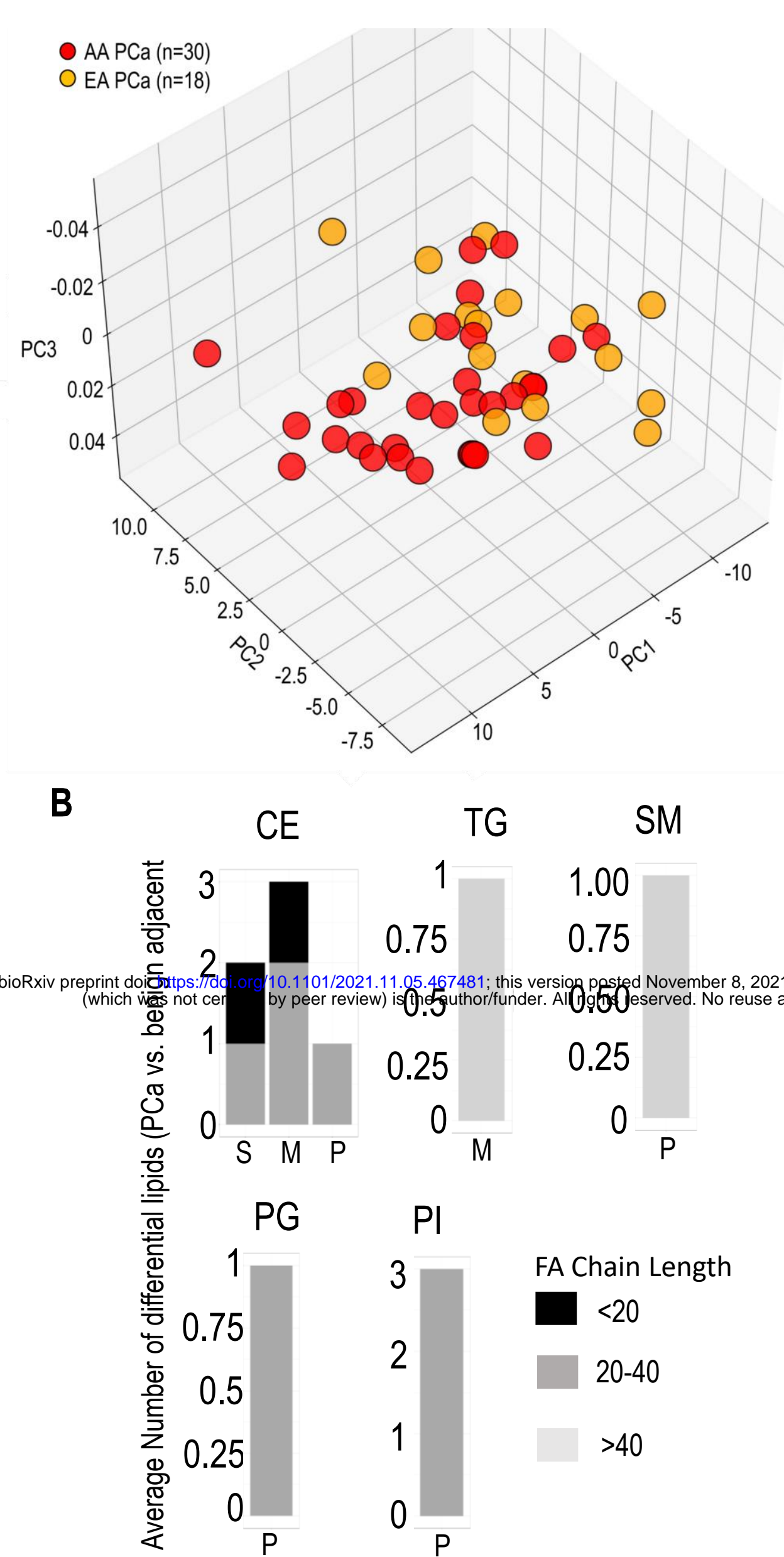

$$
\begin{aligned}
& 3 \\
& 2 \\
& 1 \\
& 0
\end{aligned}
$$

PI

TG SM

1.00

$\begin{array}{rr}1 & 1.00 \\ 0.75 & 0.75\end{array}$ 0.25

0

0

FA Chain Length

$<20$

20-40

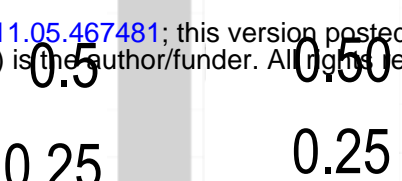

C
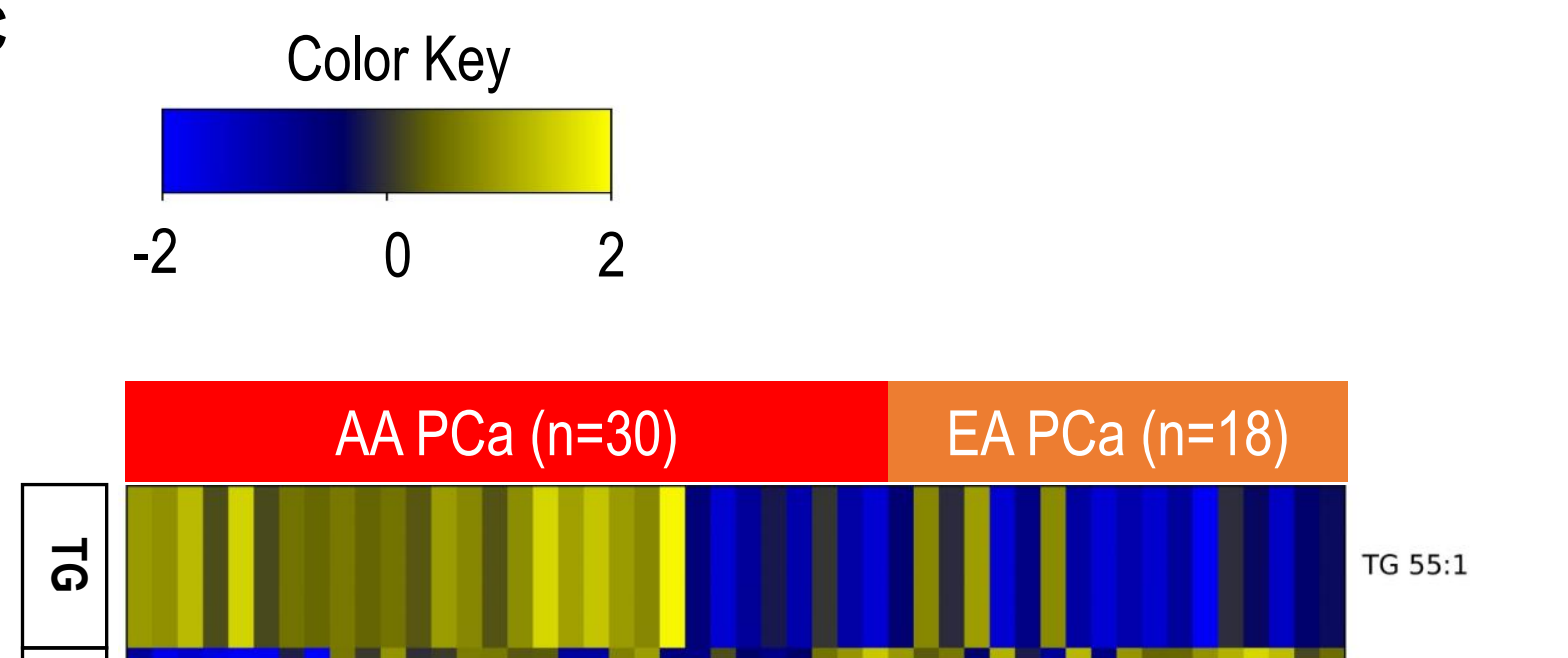

里

ำ
므

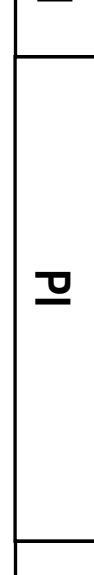

ฉั

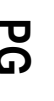

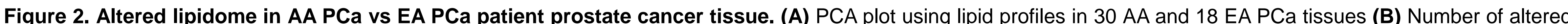

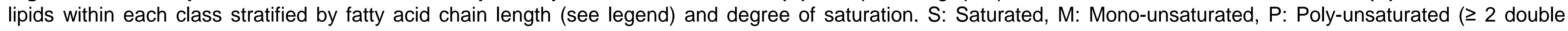

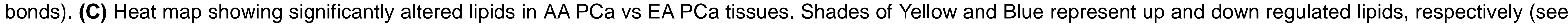

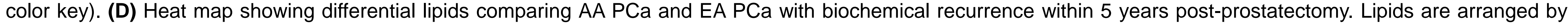

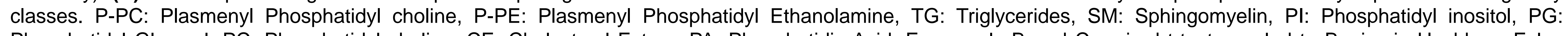

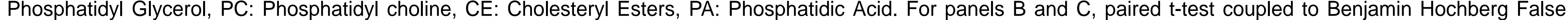

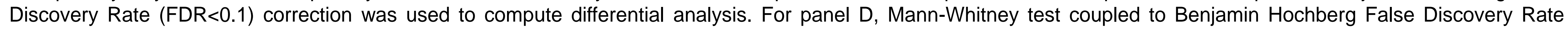
$($ FDR $<0.35)$ correction was used to compute differential analysis. Lipids that were altered at least 2 -fold with a FDR corrected $p$-value of $P<0.05$ are shown. 


\begin{tabular}{|c|c|c|}
\hline Variable & $\begin{array}{c}\text { African American } \\
(\mathbf{n}=\mathbf{3 0})\end{array}$ & $\begin{array}{c}\text { European American } \\
(\mathbf{n = 1 8})\end{array}$ \\
\hline \multirow{2}{*}{ Gleason Grade } & $\operatorname{Low}(\leq 6): 20$ & Low $(\leq 6): 6$ \\
\cline { 2 - 3 } & High $(\geq 7): 10$ & High $(\geq 7): 12$ \\
\hline Recurrence & 5 & 5 \\
\hline No Recurrence & 10 & 5 \\
\hline West African, $\hat{\mathbf{y}}^{*}$ & $0.8 \pm 0.1$ & $0.02 \pm 0.02$ \\
\hline European, $\hat{\mathbf{y}}^{*}$ & $0.2 \pm 0.1$ & $0.02 \pm 0.02$ \\
\hline Native American, $\hat{\mathbf{y}}$ & $0.0 \pm 0.0$ & $0.95 \pm 0.03$ \\
\hline $\begin{array}{c}\text { Genetic Ancestry } \\
\text { Verified }(\mathbf{n})\end{array}$ & 24 & 8 \\
\hline
\end{tabular}

